\title{
1 Low Repeatability of Aversive Learning in Zebrafish (Danio
}

2 rerio)

3

4 Dominic Mason ${ }^{1}$, Susanne Zajitschek ${ }^{1,2}$ Hamza Anwer $^{1}$, Rose E O’Dea ${ }^{1}$, Daniel Hesselson ${ }^{3,4}$,

5 Shinichi Nakagawa ${ }^{1}$

$6{ }^{1}$ Evolution and Ecology Research Centre, School of Biological and Environmental Sciences,

7 University of New South Wales, Sydney 2052 NSW, Australia.

$8{ }^{2}$ School of Biological and Environmental Sciences, Liverpool John Moores University,

9 Liverpool L3 3AF, United Kingdom.

$10{ }^{3}$ Diabetes and Metabolism Division, Garvan Institute of Medical Research, Sydney, NSW,

11 Australia.

$12{ }^{4}$ St Vincent's Clinical School, UNSW, Australia, Sydney, NSW, Australia.

14 Author for correspondence: D. Mason

15 e-mail: dpmason91@gmail.com

17 Keywords. Repeatability, Learning, Zebrafish

Ethics. All procedures approved by the Garvan Animal Ethics Committee (ARA 18_18) as

19 noted in Methods.

20 Data accessibility. All data and code can be accessed at the Open Science Framework

21 : (https://osf.io/t95v3/).

22 Author Contributions. Dominic Mason: Methodology, Data Curation, Formal Analysis, Investigation, Writing - original draft preparation, Writing - review and editing,

24 Visualisation. Susanna Zajikschek: conceptualisation, methodology, Data Curation, Writing 25 - review and editing, Supervision. Hamza Anwer: Writing - review and editing. Rose

26 O'Dea: Data Curation, Writing - review and editing, Visualisation. Daniel Hesselson:

27 Resources, Writing - review and editing. Shinichi Nakagawa: Conceptualization,

28 Methodology, Software, Formal Analysis, Resources, Data Curation, Writing - review and

29 editing, Supervision, Funding acquisition

30 Competing interests. No competing interests.

31 Funding. This research was funded through an Australian Research Council Discovery grant (DP180100818) awarded to S. Nakagawa 


\section{Summary Statement}

34 Zebrafish exhibit low repeatability (intra-class correlation) in an aversive learning assay possibly due to past selection pressure exhausting genetic variance in this learning trait.

\section{Abstract}

37 Aversive learning - avoiding certain situations based on negative experiences - can

38 profoundly increase fitness in animal species. The extent to which this cognitive mechanism could evolve depends upon individual differences in aversive learning being stable through time, and heritable across generations, yet no published study has quantified the stability of individual differences in aversive learning using the repeatability statistic, $R$ (also known as the intra-class correlation). We assessed the repeatability of aversive learning by conditioning approximately 100 zebrafish (Danio rerio) to avoid a colour cue associated with a mild electric shock. Across eight different colour conditions zebrafish did not show consistent individual differences in aversive learning $(R=0.04)$. Within conditions, when zebrafish were twice conditioned to the same colour, blue conditioning was more repeatable than green conditioning ( $R=0.15$ and $R=0.02$ ). In contrast to the low repeatability estimates for aversive learning, zebrafish showed moderately consistent individual differences in colour preference during the baseline period (i.e. prior to aversive conditioning; $R \sim 0.45$ ). Overall, aversive learning responses of zebrafish were weak and variable (difference in time spent near the aversive cue $<6$ seconds per minute), but individual differences in learning ability did not explain substantial variability. We speculate that either the effect of aversive learning was too weak to quantify consistent individual differences, or directional selection might have eroded additive genetic variance. Finally, we discuss how confounded repeatability assays and publication bias could have inflated average estimates of repeatability in animal behaviour publications. 


\section{Introduction}

Animals use the cognitive process of learning, which can be defined as a change in behaviour due to past experience, to respond to the environment (Kawecki, 2010). Learning has a profound influence on survival and reproductive success (Krebs \& Davies, 1987; Skinner, 1984), and has been studied in a wide range of taxa. For example, individual learning speed has been correlated with foraging performance in bees (Raine \& Chittka, 2008) and grasshoppers (Pasquier \& Grüter, 2016); and greater cognitive capacity has been linked to higher reproductive success in magpies (Ashton et al., 2018) and male robins (Shaw et al., 2019), as well as to healthier body condition in wild primates (Huebner, Fichtel, \& Kappeler, 2018).

Animals learn through association, which is reinforced differently by positive and negative experiences (appetitive and aversive learning, respectively). Appetitive learning takes place when individuals associate a stimulus with a 'positive' event, usually a food reward stimulus, whereas in aversive learning the association is with a 'negative' event, usually a fear inducing stimulus. Failing to learn from positive experiences (appetitive learning) prevents a potential benefit (i.e. a minor opportunity cost). Failing to learn from negative experiences may yield an immediate fatal cost. Therefore, both types of learning can increase lifetime fitness and drive natural selection, but appetitive learning may be under weaker selection than aversive learning.

For traits to evolve they need heritable variation that can be subject to selection. For labile traits (i.e. traits expressed more than once over a lifetime) the consistency of individual differences in trait expression indicates potential heritability. The common approach to quantify consistent individual differences in eco-evolutionary studies is estimating the statistical index 'repeatability' ( $R$; otherwise known as the 'intra-class correlation coefficient' or ICC; Lessells \& Boag, 1987; Nakagawa \& Schielzeth, 2010). Repeatability partitions variance into within-individual (residual) and between-individual components. Biologically, the repeatability of a trait is a measure showing the amount of observed variance which is due to individuals sustaining trait differences between each other (Nakagawa \& Schielzeth, 2010), but estimates can be inflated by measurement errors and experimental confounds (Dohm, 2002; Niemelä \& Dingemanse, 2017). 
91 Generally, behavioural traits are moderately repeatable $(R=0.34$; Bell et al., 2009; cf.

Holtmann et al., 2017), with cognitive behavioural traits showing somewhat lower repeatability $(R=0.15-0.28$; Cauchoix et al. 2018). Our understanding of how natural selection shapes the evolution of cognitive traits remains poor (Boogert et al., 2018). Despite the extensive literature on aversive learning, no published study has comprehensively quantified its repeatability (but note Cauchoix et al. (2018) includes three unpublished studies with some measures of aversive learning). To reduce this knowledge gap, we quantify the repeatability of aversive learning behaviour in zebrafish (Danio rerio), a popular model organism in cognitive science (Gerlai, 2016; Norton \& Bally-Cuif, 2010). Zebrafish exhibit a range of distinct behaviours that can be measured in previously established assays (Fangmeier et al., 2018; Meshalkina et al., 2017).

Here, we use an avoidance conditioning assay — associating a visual cue with a mild electric shock — to thoroughly assess the repeatability of aversive learning in zebrafish. We expect individuals to consistently differ in their aversive learning speeds (i.e. separation of better and worse learners). First, we examine repeatability across different colour pairs (four different pairs with eight possible combinations: 8 measurements per individual). We expect individuals to show consistent differences in aversive learning ability and, given the estimates for appetitive learning summarised in Cauchoix et al. (2018), predict a low to moderate repeatability. Second, to examine whether a constant learning environment increases the consistency of individual differences, we examine repeatability within one colour pair (both combinations of green and blue; 3 repeated measurements per individual for each colour).

\section{Methods}

\section{Zebrafish population}

116 Adult wildtype zebrafish were bred and maintained at the Garvan Institute of Medical

117 Research in Sydney, Australia. Fish were housed in 3.5L Tecniplast ZebTEC tanks

118 (maximum of 24 fish per 3.5L tank) under standard laboratory conditions $\left(\sim 28^{\circ} \mathrm{C} ; \sim \mathrm{pH} 7.5\right.$;

$119 \sim 1000 \mu$ s conductivity; 12/12h from 7:30 light/dark rotation) and fed live Artemia salina 120 nauplii twice a day and commercially available fish food once per day (O.range GROW-L). 
122 We marked juvenile fish for individual identification at around 90 days post-fertilisation with

123 coloured tags (red, brown, purple, black, white, yellow, orange, pink, or green). For marking,

124 fish were anesthetised in a tricaine solution ( $4.2 \mathrm{ml}$ of $0.4 \%$ in $100 \mathrm{ml}$ of system water) for 20

125 seconds before being injected with Visible Implant Elastomer tags (VIE, Northwest Marine

126 Technologies, Inc.; Shaw Island, Washington, United States). We injected fish twice (unless

127 one mark was blank), one on either side of the dorsal fin (Hohn \& Petrie-Hanson, 2013).

128 Among these marked fish, we used a total of 103 zebrafish with approximately equal sex ratios kept in 4 tanks of 24 individuals (12 males, 12 females) for both experiments. At any

130 one time during the experiments, the same 96 fish were used, but to compensate for death,

131 illness or experimenter error, seven fish were replaced by seven new fish over the three

132 month study. Due to incomplete data for zebrafish size (described below) the across

133 conditions and within conditions analyses included 93 and 94 zebrafish, respectively. The

134 Garvan Animal Ethics Committee approved all procedures described above and experiments

135 described below (ARA 18_18). Further, Garvan veterinarians oversaw fish welfare associated with aversive learning prior to our pilot tests.

\section{Experimental Design}

139 Aversive Learning Assay

140 We used an avoidance conditioning method to quantify aversive learning in a simple, 141 automated assay (Brock et al., 2017; Fontana et al., 2019). We ran all assays using four 142 Zantiks AD units (Zantiks Ltd., Cambridge, UK; see Supplementary Figures 1 and 2). The

143 units employed infrared tracking using an integrated computer to record fish movement and

144 collect data. In the assay, a visual cue (colour or pattern) was associated with a negative

145 stimulus (brief mild electric shock; 7V DC 80ms), which motivated fish to avoid the

146 associated visual cue. We then measured the extent of avoidance (i.e. time spent away from

147 the cue associated with an electric shock) compared to the baseline preference to quantify

148 aversive learning (association response). We based our initial assay parameters (e.g., the 149 acclimation period, voltage, etc) on previous research (Brock et al., 2017), and subsequently

150 modified the parameters based on the outcomes of pilot tests (see Supplementary

151 Information).

153 Before each assay we individually placed fish into one of four lanes within rectangular tanks

154 (see Figure 1A). For the assay, we exposed the fish to four stages; (i) Acclimation: we 
155 habituated the fish to isolation in a novel environment over a 30-minute acclimation period

156 (Figure 1B); (ii) Baseline: the tank was visually split into two even zones via the colour

157 displaying screen at the bottom of the tank (Figure 1C). One of these two colours would later

158 become conditioned with the mild electric shock (CS+), the other colour remained

159 unconditioned (CS-). Here, the position of the colours (left or right) automatically switched

160 every five minutes for a period of 30-minutes and we recorded zebrafish preference for the

161 CS+ to obtain a baseline preference before conditioning; (iii) Conditioning: first, the CS+

162 (visual cue associated with shock) was displayed across the entire screen for 1.5 seconds then

163 immediately afterwards paired with the US (mild electric shock) to condition the fish to an

164 aversive experience. Second, the CS- (visual cue not associated with shock) covered the

165 screen for 8.5 seconds (Figure 1D). This phase was repeated nine times, sufficient for fish

166 learning to avoid the CS+; and, (iv) Probe: akin to the baseline period, the tank was split into

167 two even zones (left or right) depicted by different visual cues. We tracked fish movement

168 and recorded fish preference for the visual cue associated with the shock (CS+) over 5

169 minutes. During this time, the visual cues switched every minute (see Figure 1E). Probe CS+

170 preference was used in comparison to baseline CS+ preferences to quantify learning. We

171 used only 2 minutes out of the 5-minute probe time since we determined in our observations

172 (see Supplementary Figure $5 \& 6$ ) a clear decrease in learning response. This probe length is

173 similar to other studies Brock et al. (2017) use a 2-minute probe and Fontana et al., (2019)

174 use a 1 minute probe. 

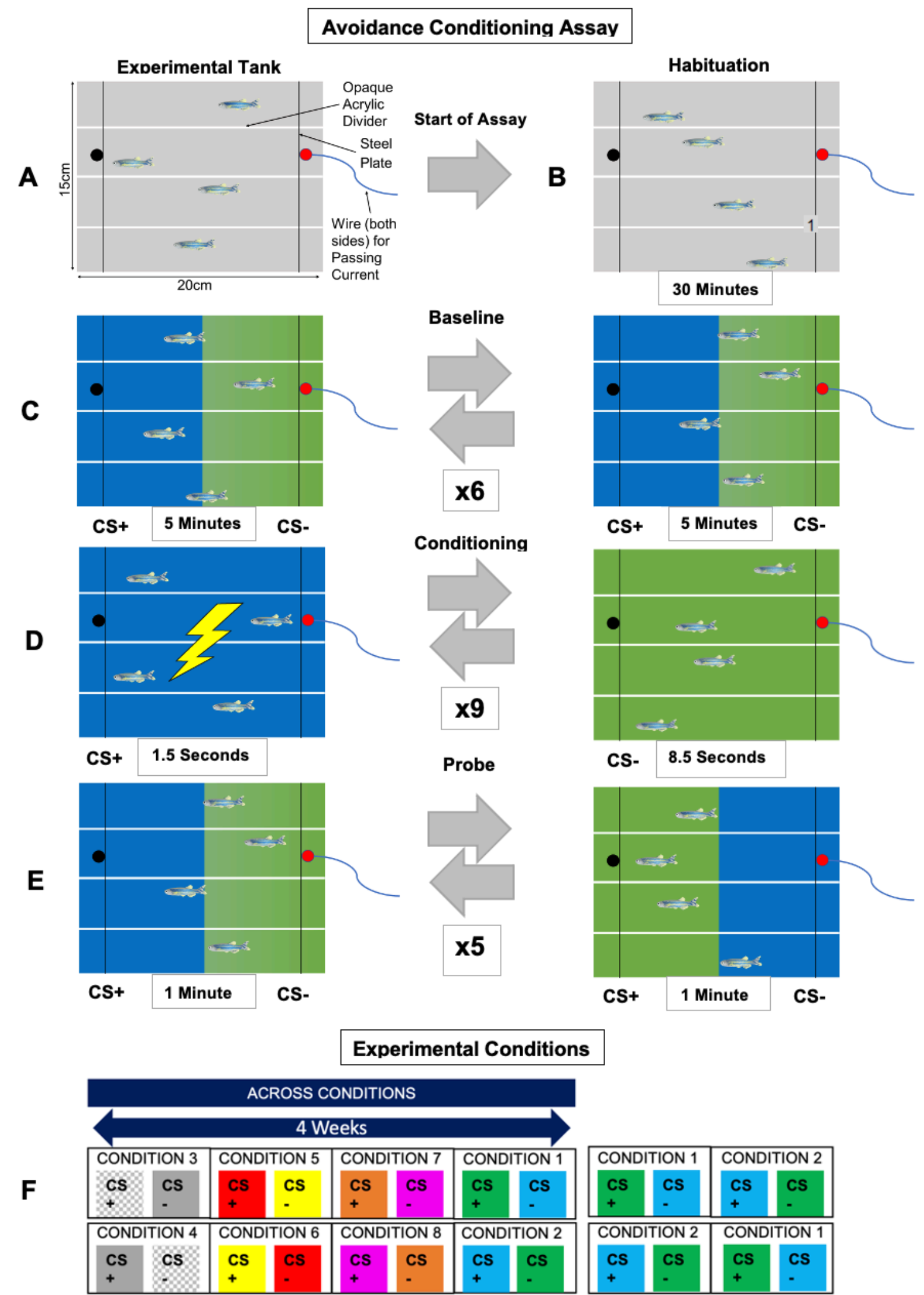

WITHIN CONDITIONS 


\section{$177 \quad$ Figure 1}

178 Colour conditions and aversive learning assay: (A) zebrafish are placed in the experimental

179 tanks and (B) acclimated to the novel environment for 30-minutes; (C) in a 30-minute

180 baseline period, initial CS \pm preference is established; (D) during the conditioning phase, fish

181 are presented the CS+, then immediately subjected to a mild electric shock; and (E) in a 5-

182 minute probe phase, learning is determined by fish spending less time in the CS+ when

183 compared to the baseline. (F) Each condition is a combination of two visual cues (zones), one

184 conditioned to a mild electric shock (CS+), the other is not (CS-). Across conditions eight

185 colour conditions and eight sessions (each session is represented by a white box). Within

186 conditions: two colour conditions and four sessions (in addition to two sessions in

187 Experiment 1).

\section{Experimental Conditions}

190 We used a range of colour conditions to test aversive learning. Each condition was comprised of two visual cues, one aversive and one control (CS+ paired with CS-) (Figure 1F). We selected different colour combinations to use as visual cues for the zebrafish, which had either been worked in pre-existing assays or were reported to evoke a clear colour preference

194 (Brock et al., 2017; Roy et al., 2019). As a result, we chose seven colours (green, blue, grey, orange, magenta, red, yellow) and 1 pattern (check; hereafter, this pattern is also referred to as a 'colour' with the others). We used four visual cue combinations ('Check/Grey',

197 'Green/Blue', 'Red/Yellow', 'Magenta/Orange') and their reverse ('Grey/Check',

198 'Blue/Green', 'Yellow/Red', 'Orange/Magenta') for a total of eight conditions. For example,

199 the 'Check/Grey' condition used check pattern as the CS+ (cue associated with shock) and

200 grey colour as the CS- (control cue); the 'Grey/Check' condition used grey colour as the CS+ and check pattern as the CS-, and so on.

Prior to the experiment, we assigned fish into quartets (four fish that underwent trials within the same Zantiks unit/assay tank simultaneously) that systematically rotated between trials. The balanced design accounted for three potential confounding variables: the time of day (quartet rotated), Zantiks unit (quartet rotated), and lane position (individual within quartet rotated). We estimated repeatability in two different situations (across conditions and within a single condition). Across conditions, we ensured fish experienced trials from all four colour pairs before subjecting them to their exact reverse four conditions (with trials conducted over 
four weeks in June and July 2019). We included this form of reverse learning to negate memory of the CS+ colour between trials, which may impact both baseline and probe colour

212 preference. Within conditions, each zebrafish underwent trials in the 'Blue/Green' and

213 'Green/Blue' conditions a further two times (over two weeks in September 2019).

\section{Fish Size Measuring}

216 We took photos of each fish approximately one week after across-conditions trials and

217 another set of photos approximately one week after within-conditions trials. We captured top

218 down photos of live fish and measured fish in ImageJ (Schindelin et al., 2015). We used fish

219 length (standard length) and width (at widest part of body) to calculate the ellipsoid size of

220 the fish by using $\pi\left(\left(\frac{\text { fish length }}{2}\right) \times\left(\frac{\text { fish width }}{2}\right)\right)$. This controlled for a potential size effect

221 resulting from loss of penetrance and effectiveness of the mild electric shock due to larger

222 body size.

\section{Data Processing and Analysis}

225 All data processing and analyses were conducted in the $R$ computing environment (version

226 4.0.2; R Core Team, 2019). Linear mixed models were run using the lme4 package (version

227 1.1.21; Bates et al., 2014) in conjunction with the lmerTest package (version 3.1.2;

228 Kuznetsova, Brockhoff, \& Christensen, 2017), that provides Satterthwaite's degrees of

229 freedom correction. We obtained repeatability values via the $r p t R$ package (version 0.9.22;

230 Stoffel et al., 2017) that uses the lme4 pacakge to run mixed models. Based on visual assessments of residual distributions, assumptions of normality and constant variance were not clearly violated. The Zantiks units recorded time spent in each CS zone, total distance travelled and how often fish changed zones. All code, and the raw and processed data, are available at: https://osf.io/t95v3/. We deemed our results statistically significant at the alpha $=0.05$ level (or when $95 \%$ confidence intervals did not overlap zero).

\section{Quantifying Aversive Learning}

238 We determined learning by the difference in time that fish spent in the CS+ before and after the aversive experience. To analyse learning across all the sessions included in this study, we used the time difference ('difference' = time spent in the CS + during baseline - time spent in the $C S+$ during probe) as the response variable in a linear mixed-effects model (LMM) via the lmer function in the lme4 package. We fitted individual 'fish ID' 96 levels) and 
'experimental condition ID' (8 levels, see Figure 1F) as random effects in the model. Also, we included the following fixed effects: (1) 'sex' (female or male) to investigate sex differences in learning, (2) 'day' since first trial, to account for time effects of sequential days on learning or learning via repeated trials (e.g., 1 being the first day and 8 being the $7^{\text {th }}$ day from the first), (3) 'fish size' to control for fish's response to conditioning which might be size dependent due to potential differences in body penetrance of a mild shock, (4) 'learning' (initial and reverse) to find if learning was affected when the CS \pm of a condition were switched in successive trials. Note that we $z$-transformed the fixed effects 'day' and 'fish size' to make the intercept meaningful and slope estimates comparable (Schielzeth, 2010).

\section{Quantifying the Repeatability of Aversive Learning}

254 We obtained enhanced agreement repeatability (hereafter referred to as repeatability) estimates by incorporating statistically significant fixed effects from the model and retaining their variance in the denominator (Nakagawa \& Schielzeth, 2010). We only fitted the random effect 'fish ID' and included 'sex' as a fixed effect. The R package $r p t R$ computes repeatability values using the within and between individual variance in linear mixed models fitted with restricted maximum likelihoods (Nakagawa \& Schielzeth, 2010). Using $r p t R$, we obtained standard errors and 95\% confidence intervals (CIs), each model set to 10,000 bootstrap samples. Following Bell (2009) and Wolak (2012), we categorised our repeatability results into low $(<0.2)$, moderate $(>0.2-<0.4)$ and high $(>0.4)$.

\section{Colour Preference and Repeatability}

An underlying assumption of our aversive learning assay was that zebrafish have the ability to discriminate between different colours. Therefore, from the baseline period (prior to aversive conditioning), we quantified underlying colour preferences (tendency to associate more heavily with one colour in a pair), and the consistency of individual differences in colour preference (i.e. repeatability of colour preference).

In each condition, preference for one colour was only compared to the other paired colour (e.g. preference for red is only relative to preference for yellow; see Figure 1F). Given we examined relative colour preference, preferences for either colour in a condition were the inverses of each other. Hence, to be able to determine colour preference for each colour, we 
'Red/Yellow' \& 'Yellow/Red'; Group 2, ‘Green/Blue' \& 'Blue/Green'; Group 3,

To analyse relative colour preference, we ran LMMs for each group of colours using across conditions data. We used baseline colour preference as the response variable 'baseline' for these models. We fitted the random effect 'fish ID' in the models (Group $1 \& 4$, 97 levels; Group $2 \&$ 3, 98 levels; levels differ because one fish died prior to completing all conditions). Further, we fitted the following fixed effects: (1) 'day' (days since first trial) to control for potential colour preference change with time, (2) 'sex' (male or female) to account for sex differences and (3) 'learning' (initial and reverse) to see the effect of reverse learning on colour preference. To determine the repeatability of colour preference, we used $r p t R$ mixedeffects models with the response variable 'baseline' to generate repeatability estimates. We did not find any fixed effects to be statistically significant, as such, they were excluded, and the colour preference models were fit with the random effect 'fish ID'.

\section{Results}

Do Zebrafish Show Appropriate Responses in an Aversive Learning Assay?

Zebrafish spent more time avoiding the CS+ following conditioning, showing evidence of learning (across conditions: female average $=3.89$ seconds per $\min , \mathrm{SE}=1.05, \mathrm{t}_{33}=3.65, P$ $<0.001 ;$ male average $=5.64$ seconds per min, $\mathrm{SE}=0.94, \mathrm{t}_{22}=5.21, P<0.001 ;$ Figure $\left.2 \mathrm{~B}\right)$. significant (1.75 seconds per min, $\left.\mathrm{SE}=0.90, \mathrm{t}_{108}=1.93, P=0.055\right)$. Reverse learning had a non-significant slight negative effect $\left(-1.11\right.$ seconds per min, $\mathrm{SE}=1.03, \mathrm{t}_{1008}=-1.07, P=$ 0.281). All other fixed effects did not significantly impact learning (see Supplementary Table 3 for all model outputs).

Is Aversive Learning Repeatable Across and Within Conditions?

303 We found very low repeatability across the eight different conditions $(R=0.04,95 \% \mathrm{CI}$ [0.001 - 0.097], Figure 3B). Within conditions, the repeatability (point-estimate) of the 'Green/Blue' condition was even lower than the across-condition estimate $(R=0.02,95 \% \mathrm{CI}$ [0 - 0.153]), while repeatability was higher in the 'Blue/Green' $(R=0.15,95 \% \mathrm{CI}[0.023$ - 
309 Do Zebrafish Display Colour Preferences and is Preference Repeatable?

310 Zebrafish showed strong relative colour preference in all four conditions (see Figure 2B). In

311 addition, fish exhibited repeatable relative colour preferences which were highly consistent

312 across all four conditions (Figure 3A; Grey: $R=0.45,95 \%$ CI [0.276 - 0.607]; Green: $R=$

$3130.45,95 \%$ CI [0.278 - 0.604]; Red: $R=0.43,95 \%$ CI [0.250 - 0.584]; Orange: $R=0.46 ; 95 \%$

314 CI [0.283 - 0.605]; see Supplementary Table 1 and 2 for all repeatability estimates).

315

316 


\section{Baseline Colour Preferences}

(A)

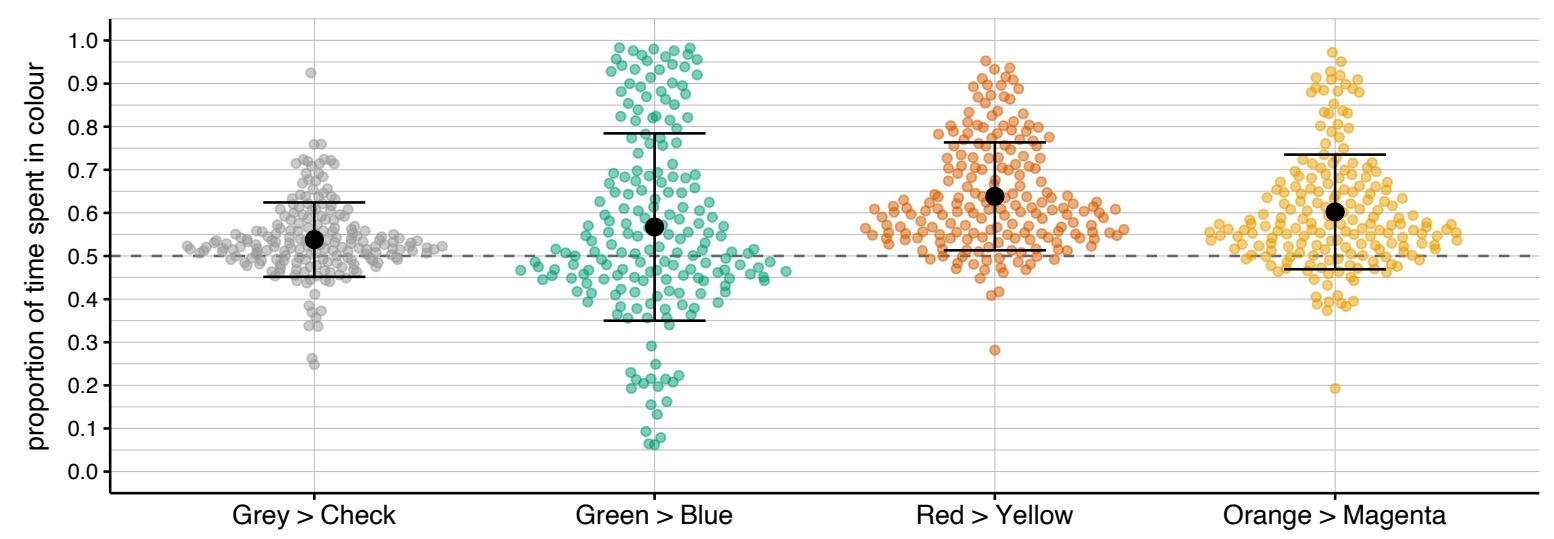

Aversive Learning

(B)

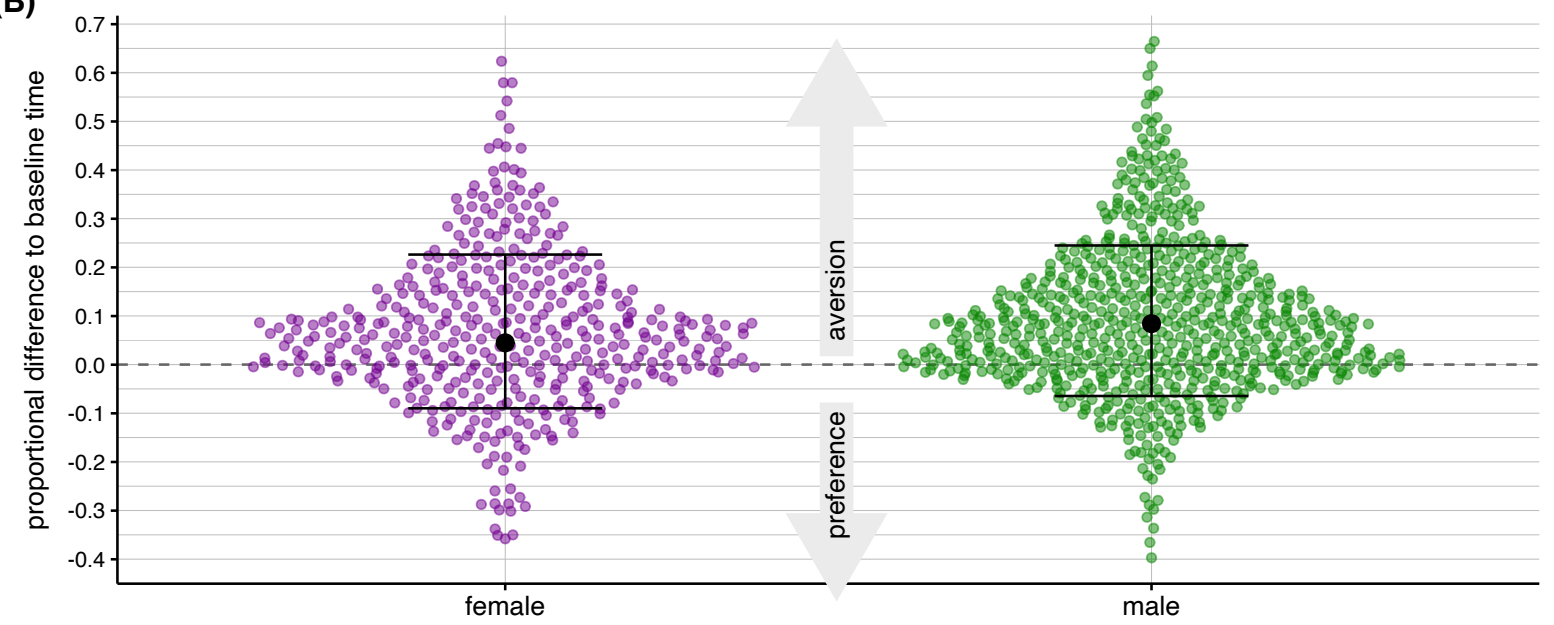

\section{Figure 2}

320 Violin plots for colour preferences and aversive learning. Smaller coloured points depict

321 individual trials. Larger black points and error bars depict means and standard deviations of

322 observations. (A) The top panel shows the tendency of zebrafish to favour one colour in a

323 pair during the baseline period (i.e. before administration of electric shocks). The dashed

324 horizontal line at 0.5 represents no colour preference (i.e. spending 30 seconds in each colour

325 zone). (B) The bottom panel shows means and variation in aversive learning, split by sex

326 (female $=$ purple; male $=$ green $)$ when all the session data is combined. Points above the line

327 at zero depict trials in which zebrafish spent less time in the aversive stimulus colour in the

328 probe period (the colour associated with an electric shock) relative to the baseline period (i.e.

329 aversive learning). 
(A)

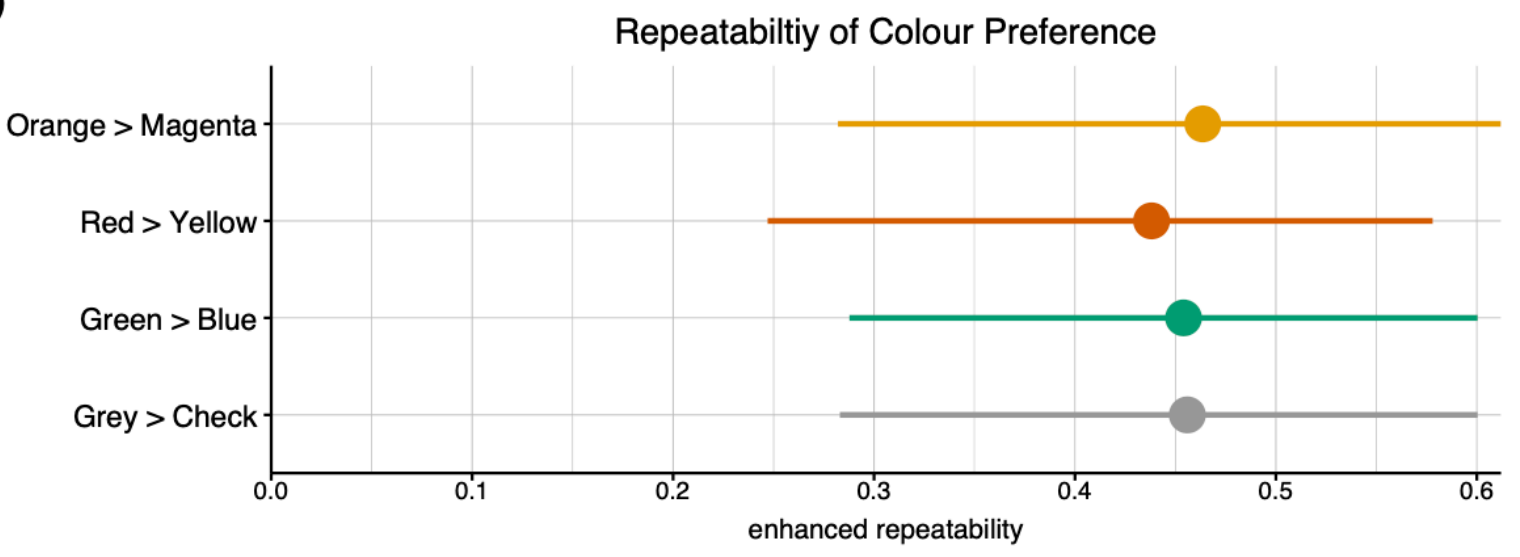

(B)

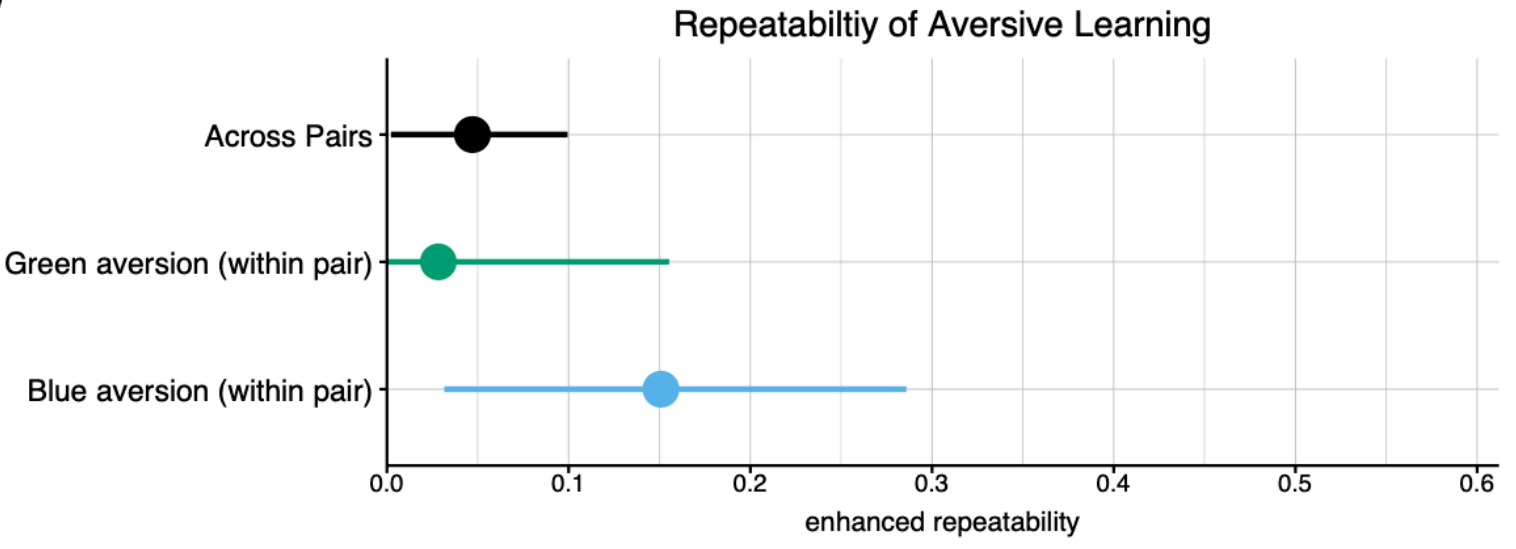

\section{Figure 3}

333 Repeatability of colour preference and aversive learning in zebrafish. Points and whiskers

334 represent means and 95\% confidence intervals via parametric bootstrapping. (A) Zebrafish

335 show consistent individual differences in colour preferences (variation depicted in Figure 2).

336 (B) Zebrafish show somewhat consistent individual differences in aversive learning within

337 the Blue/Green pair, but not within the Green/Blue pair or across all colour combinations. 


\section{Discussion}

340 We investigated aversive learning in zebrafish and quantified repeatability in two scenarios.

341 We first tested if fish displayed stable individual differences across different learning

342 environments, equivalent to methods investigating 'animal personality' (i.e. consistent

343 differences over time and contexts; Sih et al., 2004). We found negligible repeatability in

344 aversive learning across conditions, despite individuals being able to discriminate between

345 colours (as measured by a moderate repeatability of colour preferences). Then, we examined

346 repeatability within two separate conditions, which is more consistent with the idea of

347 'pseudo-repeatability' (where consistency is inflated due to measurements under an identical

348 condition; Niemelä \& Dingemanse, 2017). Within two conditions, we found negligible

349 repeatability in one condition ('Green/Blue' $R=0.02$ ), and low repeatability in the other

350 ('Blue/Green' $R=0.15$; Figure 3B). Therefore, the substantial variation in aversive learning

351 we observed was most likely driven by current (intrinsic or extrinsic) environmental factors,

352 rather than additive genetic variance or canalized developmental differences (cf. Sznajder,

353 Sabelis, \& Egas, 2012)

355 Our results are surprising, given low to moderate repeatability of behaviour and cognition reported in two meta-analyses. For behaviour generally, Bell et al (2009) reported an average repeatability of $R=0.34$. For cognitive performance, Cauchoix et al. (2018) found $R=0.15$ 0.28 , mostly based on temporal repeatability estimates from appetitive learning trials. Below we discuss four potential reasons why zebrafish in our experiment showed much less consistent individual differences in average learning compared to those previous estimates from Cauchoix et al. (2018) and Bell et al. (2009).

First, while zebrafish did demonstrate aversive learning, the effect was small, and in many trials, individuals did not seem to avoid the negative stimulus, perhaps due to not learning or quickly forgetting; on average, individuals spent 3.89 (females) and 5.64 (males) fewer seconds per minute respectively in the negatively associated colour following conditioning (Figure 2B). Low repeatability could therefore be caused by zebrafish being largely insensitive to the conditioning (i.e. bad aversive learners, or a weak assay). However, the fact that there was a population shift in the direction of aversive learning raises the question of why individuals who learnt in one trial did not maintain their performance across trials; if a particular subset of zebrafish had consistently learnt, or failed to learn, then we would have 
detected higher repeatability. Further, while the behaviour change following aversive conditioning was modest, zebrafish learnt much faster (in 1.5 minutes) compared to previous assays with appetitive training (e.g., over 20 days; Brocks et al. 2017). As far as we are aware, no studies have investigated a relationship between the strength of associative learning and the magnitude of repeatability.

Second, past selection pressures on our study population may have eroded additive genetic variance associated with aversive learning, which was not restored in the intervening generations. In the wild, aversive learning could be under strong selection (e.g. to learn to evade predators), and individuals could be selected to learn from negative experiences as quickly as possible. Indeed, aversive learning could be under stronger selection than appetitive learning, as mortality costs of negative experiences can easily exceed opportunity costs of missing positive experiences. Stronger selective pressures could explain why we found substantially lower repeatability for aversive learning compared with previous results for appetitive learning. In a similar vein, a trait more closely associated with fitness (e.g., aversive learning) tends to not be as heritable (thus, repeatable; cf. Dohm, 2002) than less fitness related traits (e.g., appetitive learning; Merilä \& Sheldon, 2000). However, we cannot be sure that whether the performance of zebrafish in our laboratory assay accurately captures their ability to aversively learn in their natural habitat. Bell et al. (2009) may have been overestimated. An inflated repeatability estimate, also known as 'pseudo-repeatability', is the result of within-individual variation being erroneously accredited to differences between individuals (Niemelä \& Dingemanse, 2017; Westneat, Hatch, Wetzel, \& Ensminger, 2011). Pseudo-repeatability occurs when the conditions between measurements are too similar (e.g., environmental conditions are unchanged or intervals between measurements are too short), and might explain why we found higher repeatability when zebrafish were measured repeatedly within a single condition ('Blue/Green'; $R=0.15$ ), compared to across eight separate conditions (although no inflation was seen in 'Green/Blue'). On closer inspection, some of studies in Cauchoix et al. (2018) and Bell et al. (2009) included testing conditions which did not change over the course of a study, similar to our within-condition estimates. Further, both Cauchoix (2018) predominately included studies with intervals under a week and Bell et al. (2009) almost all were under a year. Bell et al. (2009) reported that short intervals between measurements were 
406

407

408

409

410

411

412

413

414

415

416

417

418

419

420

421

422

423

424

425

426

427

428

429

430

431

432

433

434

435

436

signficantly associated with higher repeataibltiy values in line with pseudo-repeatability.

Relevantly, two recent studies on birdsong reported that associative learning among individuals was not repeatable between years, indicating that estimates obtained over short intervals may not be a true reflection of phenotypic constancy defined in animal personality (Soha et al., 2019; Zsebők et al., 2017).

Fourth, the meta-analytic repeatability estimates by Bell et al. (2009) might have been overestimated due to a potentially widespread publication bias in the literature reporting repeatability of behaviour (cf. Parker et al., 2016). Our across conditions repeatability estimate is markedly low in comparison to that of general behaviour founded in Bell et al. (2009; $R=0.34)$ that only included published studies. Cauchoix et al. (2018) included many unpublished datasets $(n=38)$ compared to published datasets $(n=6)$; they mentioned that their unpublished datasets produced, overall, a lower repeatability estimate than that of the published studies. This finding is consistent with the pattern that larger effect sizes are more likely to be published. It is possible that publication bias has further contributed to an inflation of the overall repeatability estimates in the published literature. However, recent studies are increasingly reporting non-significant and low repeatability (e.g., Reichert et al., 2020; Vernouillet \& Kelly, 2020). Therefore, an updated future meta-analysis may reveal a lower overall repeatability estimate in behaviour.

In conclusion, zebrafish did not show clear consistent between-individual differences in aversive learning. The low repeatability could potentially indicate that strong past selection pressure has almost driven aversive learning to fixation, because of the vital importance to learn to avoid danger. In addition, many researchers may have unknowingly included confounded pseudo-repeatability results in their studies. In turn, inflating published repeatability estimates and presenting the repeatability of behaviour and learning-associated behaviour higher than the 'true' repeatability of behaviour. Further, a bias to withhold nonsignificant findings from publishing may have exacerbated this inflation in the literature. We contend that these issues can be diminished in future behavioural research by controlling for confounding effects and reporting every estimate of behavioural traits, whether repeatable or not. 


\section{Acknowledgement}

438 We are grateful for the staff at the Biological Testing Facility, Garvan Institute of Medical 439 Research for their support and husbandry of zebrafish. This study was supported by ARC 440 (Australian Research Council) Discovery grant (DP180100818). No competing interests 441 declared.

442

443

444

445

446

447

448

449

450

451

452

453

454

455

456

457

458

459

460

461

462

463

464

465

466

467

468

\section{References}

Ashton, B. J., Ridley, A. R., Edwards, E. K., \& Thornton, A. (2018). Cognitive performance is linked to group size and affects fitness in Australian magpies. Nature, 554(7692), 364-367. https://doi.org/10.1038/nature25503

Baker, M. R., \& Wong, R. Y. (2019). Contextual fear learning and memory differ between stress coping styles in zebrafish. Scientific Reports, 9(1). https://doi.org/10.1038/s41598019-46319-0

Bates, D., Mächler, M., Bolker, B., \& Walker, S. (2014). Fitting linear mixed-effects models using lme4. ArXiv Preprint ArXiv: 1406.5823.

Bell, A. M., Hankison, S. J., Laskowski, K. L., Bell, Alison M, Shala J. Hankison, and K. L. L., Bell, A. M., Hankison, S. J., \& Laskowski, K. L. (2009). The repeatability of behaviour: a meta-analysis. Animal Behaviour, 77(4), 771-783. https://doi.org/10.1016/j.anbehav.2008.12.022.Bell

Boogert, N. J., Madden, J. R., Morand-Ferron, J., \& Thornton, A. (2018). Measuring and understanding individual differences in cognition. Philosophical Transactions of the Royal Society B: Biological Sciences, 373(1756). https://doi.org/10.1098/rstb.2017.0280

Brock, A. J., Sudwarts, A., Daggett, J., Parker, M. O., \& Brennan, C. H. (2017). A fully automated computer based Skinner box for testing learning and memory in zebrafish. BioRxiv, 110478. https://doi.org/10.1101/110478

Cauchoix, M., Chow, P. K. Y. K. Y., Van Horik, J. O. O., Atance, C. M. M., Barbeau, E. J. J., Barragan-Jason, G., ... Morand-Ferron, J. (2018). The repeatability of cognitive performance: A meta-analysis. Philosophical Transactions of the Royal Society B: Biological Sciences, 373(1756). https://doi.org/10.1098/rstb.2017.0281

Dohm, M. R. R. (2002). Repeatability estimates do not always set an upper limit to heritability. Functional Ecology, 16(2), 273-280. https://doi.org/10.1046/j.13652435.2002.00621.x

Fangmeier, M. L., Noble, D. W. A., O’Dea, R. E., Usui, T., Lagisz, M., Hesselson, D., \& 
Nakagawa, S. (2018). Computer Animation Technology in Behavioral Sciences: A Sequential, Automatic, and High-Throughput Approach to Quantifying Personality in Zebrafish (Danio rerio). Zebrafish, 15(2), 206-210. https://doi.org/10.1089/zeb.2017.1532

Fontana, B D, Cleal, M., Clay, J. M., \& Parker, M. O. (2019). Zebrafish (Danio rerio) behavioral laterality predicts increased short-term avoidance memory but not stressreactivity responses. Animal Cognition. https://doi.org/10.1007/s10071-019-01296-9

Fontana, Barbara Dotto, Cleal, M., Clay, J., \& Parker, M. O. (2019). Zebrafish (Danio rerio) behavioral laterality predicts increased short-term avoidance memory but not stressreactivity responses. BioRxiv, 565309. https://doi.org/10.1101/565309

Gerlai, R. (2016). Learning and memory in zebrafish (Danio rerio). Methods in Cell Biology, 134, 551-586. https://doi.org/10.1016/bs.mcb.2016.02.005

Hohn, C., \& Petrie-Hanson, L. (2013). Evaluation of visible implant elastomer tags in zebrafish (Danio rerio). Biology Open, 2(12), 1397-1401. https://doi.org/10.1242/bio.20136460

Holtmann, B., Lagisz, M., \& Nakagawa, S. (2017). Metabolic rates, and not hormone levels, are a likely mediator of between-individual differences in behaviour: a meta-analysis. Functional Ecology, 31(3), 685-696. https://doi.org/10.1111/1365-2435.12779

Huebner, F., Fichtel, C., \& Kappeler, P. M. (2018). Linking cognition with fitness in a wild primate: Fitness correlates of problem-solving performance and spatial learning ability. Philosophical Transactions of the Royal Society B: Biological Sciences, 373(1756). https://doi.org/10.1098/rstb.2017.0295

Kaneko, S., Masuda, R., \& Yamashita, Y. (2019). Memory retention capacity using two different training methods, appetitive and aversive learning, in juvenile red sea bream Chrysophrys major. Journal of Fish Biology, 94(2), 231-240. https://doi.org/10.1111/jfb.13876

Kawecki, T. J. (2010). Evolutionary ecology of learning: Insights from fruit flies. Population Ecology, 52(1), 15-25. https://doi.org/10.1007/s10144-009-0174-0

Kenney, J. W., Scott, I. C., Josselyn, S. A., \& Frankland, P. W. (2017). Contextual fear conditioning in zebrafish. Learning and Memory, 24(10), 516-523. https://doi.org/10.1101/1m.045690.117

Krebs, J. R., \& Davies, N. B. (1987). An introduction to behavioural ecology, 2nd ed. In An introduction to behavioural ecology, 2nd ed. Sunderland, MA, US: Sinauer Associates. Kuznetsova, A., Brockhoff, P. B., \& Christensen, R. H. B. (2017). 1merTest Package: Tests in 

https://doi.org/10.18637/jss.v082.i13

Lessells, C. M., \& Boag, P. T. (1987). Unrepeatable Repeatabilities: A Common Mistake. The Auk, 104(1), 116-121. https://doi.org/10.2307/4087240

Merilä, J., \& Sheldon, B. C. (2000). Lifetime reproductive success and heritability in nature. American Naturalist, 155(3), 307-310. https://doi.org/10.2307/3078867

Meshalkina, D. A., Kizlyk, M. N., Kysil, E. V., Collier, A. D., Echevarria, D. J., Abreu, M. S., ... Kalueff, A. V. (2017). Understanding zebrafish cognition. Behavioural Processes, Vol. 141, pp. 229-241. https://doi.org/10.1016/j.beproc.2016.11.020

Nakagawa, S., \& Schielzeth, H. (2010, November). Repeatability for Gaussian and nonGaussian data: A practical guide for biologists. Biological Reviews, Vol. 85, pp. 935956. https://doi.org/10.1111/j.1469-185X.2010.00141.X

Namekawa, I., Moenig, N. R., \& Friedrich, R. W. (2018). Rapid olfactory discrimination learning in adult zebrafish. Experimental Brain Research, 236(11), 2959-2969. https://doi.org/10.1007/s00221-018-5352-x

Niemelä, P. T. T., \& Dingemanse, N. J. J. (2017). Individual versus pseudo-repeatability in behaviour: Lessons from translocation experiments in a wild insect. Journal of Animal Ecology, 86(5), 1033-1043. https://doi.org/10.1111/1365-2656.12688

Norton, W., \& Bally-Cuif, L. (2010). Adult zebrafish as a model organism for behavioural genetics. BMC Neuroscience, Vol. 11. https://doi.org/10.1186/1471-2202-11-90 Nakagawa, S. (2016). Transparency in Ecology and Evolution: Real Problems, Real Solutions. Trends in Ecology and Evolution, Vol. 31, pp. 711-719. https://doi.org/10.1016/j.tree.2016.07.002

Pasquier, G., \& Grüter, C. (2016). Individual learning performance and exploratory activity are linked to colony foraging success in a mass-recruiting ant. Behavioral Ecology, 1(1), arw079. https://doi.org/10.1093/beheco/arw079

Raine, N. E., \& Chittka, L. (2008). The correlation of learning speed and natural foraging success in bumble-bees. Proceedings of the Royal Society B: Biological Sciences, 275(1636), 803-808. https://doi.org/10.1098/rspb.2007.1652 Quinn, J. L. (2020). Multiple factors affect discrimination learning performance, but not between-individual variation, in wild mixed-species flocks of birds. 

https://doi.org/10.1038/s41598-019-51145-5

Schielzeth, H. (2010). Simple means to improve the interpretability of regression coefficients. Methods in Ecology and Evolution, 1(2), 103-113. Retrieved from http://doi.wiley.com/10.1111/j.2041-210X.2010.00012.x

Schindelin, J., Rueden, C. T., Hiner, M. C., \& Eliceiri, K. W. (2015). The ImageJ ecosystem: An open platform for biomedical image analysis. Molecular Reproduction and Development, 82(7-8), 518-529. https://doi.org/10.1002/mrd.22489

Shaw, R. C., Mackinlay, R. D., Clayton, N. S., Burns, K. C., Shaw, R. C., Mackinlay, R. D., ... Burns, K. C. (2019). Memory Performance Influences Male Reproductive Success in a Wild Bird Memory Performance Influences Male Reproductive Success in a Wild Bird. Current Biology, 29(9), 1498-1502.e3. https://doi.org/10.1016/j.cub.2019.03.027

Sih, A., Bell, A., \& Johnson, J. C. (2004, July). Behavioral syndromes: An ecological and evolutionary overview. Trends in Ecology and Evolution, Vol. 19, pp. 372-378. https://doi.org/10.1016/j.tree.2004.04.009

Skinner, B. F. (1984). THE EVOLUTION OF BEHAVIOR. Journal of the Experimental Analysis of Behavior, 41(2), 217-221. https://doi.org/10.1901/jeab.1984.41-217

Soha, J. A., Peters, S., Anderson, R. C., Searcy, W. A., \& Nowicki, S. (2019). Performance on tests of cognitive ability is not repeatable across years in a songbird. Animal Behaviour. https://doi.org/10.1016/j.anbehav.2019.09.020

Stoffel, M. A., Nakagawa, S., \& Schielzeth, H. (2017). rptR: repeatability estimation and variance decomposition by generalized linear mixed-effects models. Methods in Ecology and Evolution, 8(11), 1639-1644. https://doi.org/10.1111/2041-210X.12797

Sznajder, B., Sabelis, M. W., \& Egas, M. (2012). How Adaptive Learning Affects Evolution: Reviewing Theory on the Baldwin Effect. Evolutionary Biology, 39(3), 301-310. https://doi.org/10.1007/s11692-011-9155-2

Team, R. C. (2017). R: A Language and Environment for Statistical Computing. Retrieved from http://www.r-project.org/

Thomson, H. R., Lamb, S. D., Besson, A. A., \& Johnson, S. L. (2020). Long-term repeatability of behaviours in zebrafish (Danio rerio). Ethology, (October 2019), 803811. https://doi.org/10.1111/eth.13038

Vernouillet, A., \& Kelly, D. M. (2020). Individual exploratory responses are not repeatable across time or context for four species of food-storing corvid. Scientific Reports, 10(1), 1-11. https://doi.org/10.1038/s41598-019-56138-y 
571 Westneat, D. F., Hatch, M. I., Wetzel, D. P., \& Ensminger, A. L. (2011). Individual variation in parental care reaction norms: Integration of Personality and plasticity. American Naturalist, 178(5), 652-667. https://doi.org/10.1086/662173

574 Wolak, M. E. M. E. E., Fairbairn, D. J. D. J. J., \& Paulsen, Y. R. Y. R. R. (2012). Guidelines for estimating repeatability. Methods in Ecology and Evolution, 3(1), 129-137. https://doi.org/10.1111/j.2041-210X.2011.00125.x

Zsebők, S., Herczeg, G., Blázi, G., Laczi, M., Nagy, G., Szász, E., ... Garamszegi, L. Z. (2017). Short- and long-term repeatability and pseudo-repeatability of bird song: sensitivity of signals to varying environments. Behavioral Ecology and Sociobiology, 


\section{Supplementary Information (SI)}

2

3 The Repeatability of Aversive Learning in Zebrafish (Danio Rerio)

4

5 Dominic Mason ${ }^{1}$

$7 \quad{ }^{1}$ Evolution \& Ecology Research Centre, School of Biological, Earth and Environmental

8 Sciences, University of New South Wales, Sydney, NSW, Australia. 


\section{Supplementary Methods}

\section{Zantiks Experimental Units}

14 We used Zantiks AD fully automated units to conduct our behavioural experiments (Zantiks

15 Ltd., Cambridge, UK; Supplementary Figure 1). The design enabled comprehensive standardised cognitive assays on zebrafish. The boxes' capabilities include infrared tracking, a stimulus screen, feeding mechanisms, removable tanks with modifiable inserts, an in-built computer, console interface and video recording. They were well equipped to conduct simple experimental manipulation and provide a range of stimuli (colours, patterns or images) to measure behavioural responses.

During experiments, we placed portable tanks (length $20 \mathrm{~cm}$ : height $14 \mathrm{~cm}$ : width $15 \mathrm{~cm} ; 2.6 \mathrm{~L}$ system water; see Supplementary Figure 2, picture C) containing the fish onto the screens inside the units (see Supplementary Figure 2, picture B). We presented experimental stimuli via the screen through the transparent base of the tank. Fish location co-ordinates were tracked via an inbuilt infrared (IR) camera situated at the ceiling of the unit and another IR source underneath the screen. A basal screen enabled a near completely closed system inhibiting external disturbances.

\section{Pilot Experiments}

To find the best parameters to use in the avoidance task, we carried out numerous pilot assays. Specifically, we examined three parameters: stimulus type (colour or pattern), assay length and voltage. Our aim was to find the shortest assay length and lowest voltage suitable to yield a behavioural response from the fish. At the same time we determined which stimuli (colours) would be ideal, testing stimuli used in the literature (Brock et al., 2017) and two colours that we did not find in the literature, orange and magenta.

With guidance from previous research (Brock et al., 2017), we conducted preliminary tests to identify the lowest voltage setting required to yield an adequate learning response. We tested three settings: five, seven and nine volts, each applied nine times per trial. The seven-volt setting elicited the most fish avoidance for the CS+ in the probe stage (see Supplementary

42 Figure 4). Based on this finding we used seven volts applied nine times as the US in the conditioning phase for our experiments. 
The previously developed assay by Brock et al. (2017) comprised of 3 stages: baseline, conditioning and probe. We extensively tested the three stages to decide the optimal length for each. Akin to other studies, the baseline and conditioning stages remained the same length (Brock et al., 2017; Fontana et al., 2019). However, we lengthened the probe period from two to five minutes to provide a wider range for potential analysis. Further, we introduced an acclimation stage to ensure a consistent association response from the fish (Thomson et al., 2020). The acclimation stage is absent in some studies, although when present can range in length from 10-minutes (Baker \& Wong, 2019; Kenney et al., 2017) to over the course of multiple days (Kaneko, Masuda, \& Yamashita, 2019; Namekawa, Moenig, \& Friedrich, 2018). Following the data collected during our pilot assays, we found a 30-minute acclimation period just prior to data collection afforded the best association response.

Our pilot results indicated a steep decline in association response after two minutes in the probe period. Although these results aligned with the literature (Brock et al., 2017 2-minute probe; Fontana et al., 2019 1-minute probe) we integrated an extended probe period (the probe phase above) to verify if fish would display a similar deterioration. As expected, the fish exhibited a corresponding drop in association response after two minutes. Consequently, for our analysis we confined the extended probe period to two minutes since the ensuing deterioration may indicate memory loss or habituation to the $\mathrm{CS}+$ post conditioning or a new learning event.

\section{Supplementary Notes}

\section{Sex Differences in Repeatability}

We found males we more generally more repeatable than females (Figure S3) in aversive learning. We found this result across conditions (males, sample size $=63, R=0.06,95 \% \mathrm{CI}$ [0.007 - 0.091]; females, sample size $=46, R=0.00,95 \%$ CI [0 - 0.055]) and in the 'Blue/Green' condition (males, sample size $=62, R=0.23,95 \%$ CI [0.050 - 0.374]; females, sample size $=37, R=0.02,95 \%$ CI $[0-0.195])$. This result was not anticipated since females are reported to be more repeatable than males in behaviour (Bell et al., 2009). We found no statistically significant difference in repeatability between males and females, displayed by no overlap over zero in bootstrap distribution displayed in Supplementary Figure 4. 
77 In relative colour preference, we found males were more repeatable than female in the colours 78 red, grey and orange but not green. Since we are the first to assess the repeatability of colour 79 preference in zebrafish, we cannot compare to the literature, however, the sex differences in 80 colour preference repeatability are mostly consistent with those in the repeatability of aversive 81 learning.

82 


\section{Supplementary Tables}

\section{Supplementary Table 1}

85 Repeatability values for different conditions with bootstrapped 95\% confidence intervals. All

86 conditions display sexes mixed at the top then male and female results. Estimates with CIs

87 that do not overlap zero are presented in bold.

88

\begin{tabular}{|c|c|c|}
\hline Conditions & Repeatability $(R)$ & 95\% Confidence Interval \\
\hline Across & 0.047 & $0.007-0.091$ \\
\hline Across Male & 0.069 & $0.014-0.154$ \\
\hline Across Female & 0 & $0-0.055$ \\
\hline Green/Blue & 0.028 & $0-0.137$ \\
\hline Green/Blue Male & 0.039 & $0-0.222$ \\
\hline Green/Blue Female & 0.016 & $0-0.203$ \\
\hline Blue/Green & 0.150 & $0.023-0.308$ \\
\hline Blue/Green Male & 0.232 & $0.050-0.374$ \\
\hline Blue/Green Female & 0.022 & $0-0.195$ \\
\hline First & 0 & $0-0.189$ \\
\hline First Male & 0 & $0-0.227$ \\
\hline First Female & 0.029 & $0-0.383$ \\
\hline Second & 0.012 & $0-0.200$ \\
\hline Second Male & 0 & $0-0.000$ \\
\hline Second Female & 0.073 & $0-0.419$ \\
\hline
\end{tabular}




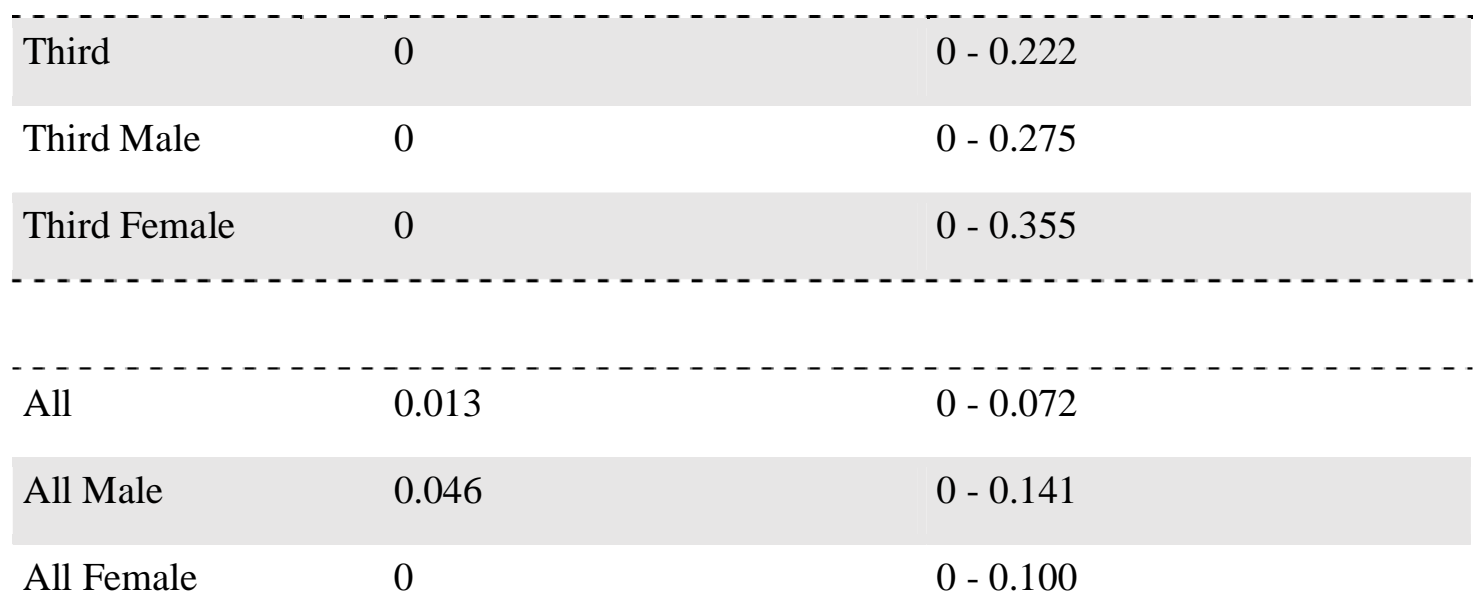

89 


\section{Supplementary Table 2}

91 Repeatability estimates of relative colour preference with bootstrapped 95\% CIs for red,

92 green, grey and orange. Male and female preference included. Estimates with CIs that do not 93 overlap zero are presented in bold.

94

\begin{tabular}{llc}
\hline \hline Colour & Repeatability $(R)$ & $95 \%$ Confidence Interval \\
\hline \hline Red & 0.438 & $0.250-0.584$ \\
Red Male & 0.492 & $0.288-0.656$ \\
Red Female & 0.331 & $0.009-0.586$
\end{tabular}

\begin{tabular}{lll}
\hline Green & 0.454 & $0.278-0.604$ \\
Green Male & 0.434 & $0.215-0.614$ \\
Green Female & 0.490 & $0.203-0.702$
\end{tabular}

$\begin{array}{lll}\text { Grey } & 0.455 & 0.276-0.607 \\ \text { Grey Male } & 0.499 & 0.309-0.657 \\ \text { Grey Female } & 0.391 & 0.056-0.635\end{array}$

\begin{tabular}{lll}
\hline Orange & 0.463 & $0.283-0.605$ \\
Orange Male & 0.519 & $0.280-0.681$ \\
Orange Female & 0.411 & $0.083-0.649$ \\
\hline
\end{tabular}




\section{Supplementary Table 3}

97 The outputs of fixed and random effects from the across conditions aversive learning mixed

98 effect model. Significant results are displayed in bold.

\begin{tabular}{|c|c|c|c|}
\hline \multirow[b]{2}{*}{ Predictors } & \multicolumn{3}{|c|}{ difference } \\
\hline & Estimates & $C I$ & $p$ \\
\hline (Intercept) & 3.90 & $1.58-6.22$ & 0.001 \\
\hline sex [male] & 1.75 & $-0.02-3.52$ & 0.053 \\
\hline session & 0.37 & $-0.71-1.45$ & 0.501 \\
\hline fish_size & 0.30 & $-0.54-1.14$ & 0.479 \\
\hline $\begin{array}{l}\text { learning_combined } \\
\text { [reverse] }\end{array}$ & -1.12 & $-3.15-0.92$ & 0.282 \\
\hline \multicolumn{4}{|l|}{ Random Effects } \\
\hline$\sigma^{2}$ & 79.76 & & \\
\hline$\tau_{00 \text { fishID }}$ & 4.43 & & \\
\hline$\tau_{00 \exp }$ & 3.23 & & \\
\hline ICC & 0.09 & & \\
\hline $\mathrm{N}_{\exp }$ & 8 & & \\
\hline $\mathrm{N}_{\text {fishID }}$ & 96 & & \\
\hline Observations & 1106 & & \\
\hline Marginal $\mathrm{R}^{2}$ / Conditional $\mathrm{R}^{2}$ & $0.007 / 0$. & .094 & \\
\hline
\end{tabular}

99 


\section{Supplementary Table 4}

101 The outputs of fixed and random effects from the across conditions red colour preference

102 mixed effect model. Significant results are displayed in bold.

103

\begin{tabular}{llll}
\hline \hline & \multicolumn{2}{l}{ BASELINE } & \\
Predictors & Estimates CI & $\mathrm{p}$ \\
\hline (Intercept) & 36.35 & $33.03-39.67$ & $<0.001$ \\
day & -1.05 & $-3.80-1.71$ & 0.457 \\
sex [male] & 1.51 & $-1.13-4.15$ & 0.262 \\
learning [reverse] & 2.05 & $-3.09-7.18$ & 0.435 \\
Random Effects & & & \\
$\sigma^{2}$ & & & \\
$\tau_{00}$ fishID & 32.10 & & \\
ICC & 24.72 & & \\
$\mathrm{~N}_{\text {fishID }}$ & 0.44 & & \\
\hline Observations & 98 & \\
Marginal R $^{2}$ / Conditional $\mathrm{R}^{2}$ & $0.014 / 0.443$ & \\
\hline & & & \\
\hline
\end{tabular}

104 


\section{Supplementary Table 5}

106 The outputs of fixed and random effects from the across conditions green colour preference

107 mixed effect model. Significant results are displayed in bold.

108

\begin{tabular}{llll}
\hline \hline & \multicolumn{2}{l}{ BASELINE } & \\
Predictors & \multicolumn{2}{l}{ Estimates CI } & $\mathrm{p}$ \\
\hline (Intercept) & 36.27 & $30.31-42.22$ & $<\mathbf{0 . 0 0 1}$ \\
day & 1.35 & $-3.52-6.23$ & 0.586 \\
sex [male] & 0.51 & $-4.13-5.16$ & 0.829 \\
learning [reverse] & -5.11 & $-14.15-3.93$ & 0.268 \\
Random Effects & & & \\
$\sigma^{2}$ & & & \\
$\tau_{00}$ fishID & 90.08 & & \\
ICC & 80.59 & & \\
$\mathrm{~N}_{\text {fishID }}$ & 0.47 & & \\
\hline Observations & 97 & \\
Marginal $^{2} /$ Conditional $\mathrm{R}^{2}$ & $0.013 / 0.479$ & \\
\hline \hline
\end{tabular}

109 


\section{Supplementary Table 6}

111 The outputs of fixed and random effects from the across conditions check colour preference

112 mixed effect model. Significant results are displayed in bold.

113

\begin{tabular}{llll}
\hline \hline & \multicolumn{2}{l}{ BASELINE } & \\
Predictors & Estimates CI & $\mathrm{p}$ \\
\hline (Intercept) & 32.81 & $30.54-35.07$ & $<\mathbf{0 . 0 0 1}$ \\
day & 0.42 & $-1.51-2.36$ & 0.667 \\
sex [male] & -0.63 & $-2.47-1.21$ & 0.503 \\
learning [reverse] & -0.26 & $-3.85-3.34$ & 0.889 \\
Random Effects & & & \\
$\sigma^{2}$ & & & \\
$\tau_{00}$ fishID & 14.61 & & \\
ICC & 12.47 & & \\
$\mathrm{~N}_{\text {fishiD }}$ & 0.46 & & \\
\hline Observations & 97 & \\
Marginal R $^{2}$ / Conditional $\mathrm{R}^{2}$ & $0.007 / 0.464$ & \\
\hline \hline
\end{tabular}

114 


\section{$115 \quad$ Supplementary Table 7}

116 The outputs of fixed and random effects from the across conditions orange colour preference

117 mixed effect model. Significant results are displayed in bold.

118

\begin{tabular}{llll}
\hline \hline & \multicolumn{2}{l}{ BASELINE } & \\
Predictors & Estimates CI & $\mathrm{p}$ \\
\hline (Intercept) & 37.08 & $33.75-40.41$ & $<0.001$ \\
day & 0.73 & $-1.95-3.41$ & 0.592 \\
sex [male] & -1.04 & $-3.86-1.78$ & 0.469 \\
learning [reverse] & -0.59 & $-5.56-4.39$ & 0.818 \\
Random Effects & & & \\
$\sigma^{2}$ & & & \\
$\tau_{00}$ fishID & 36.39 & & \\
ICC $^{2}$ & 27.15 & & \\
$\mathrm{~N}_{\text {fishiD }}$ & 0.43 & & \\
\hline Observations & 98 & \\
Marginal R $^{2}$ Conditional $\mathrm{R}^{2}$ & $0.008 / 0.432$ & \\
\hline & & & \\
\hline
\end{tabular}

119 


\section{Supplementary Figures}

121

122

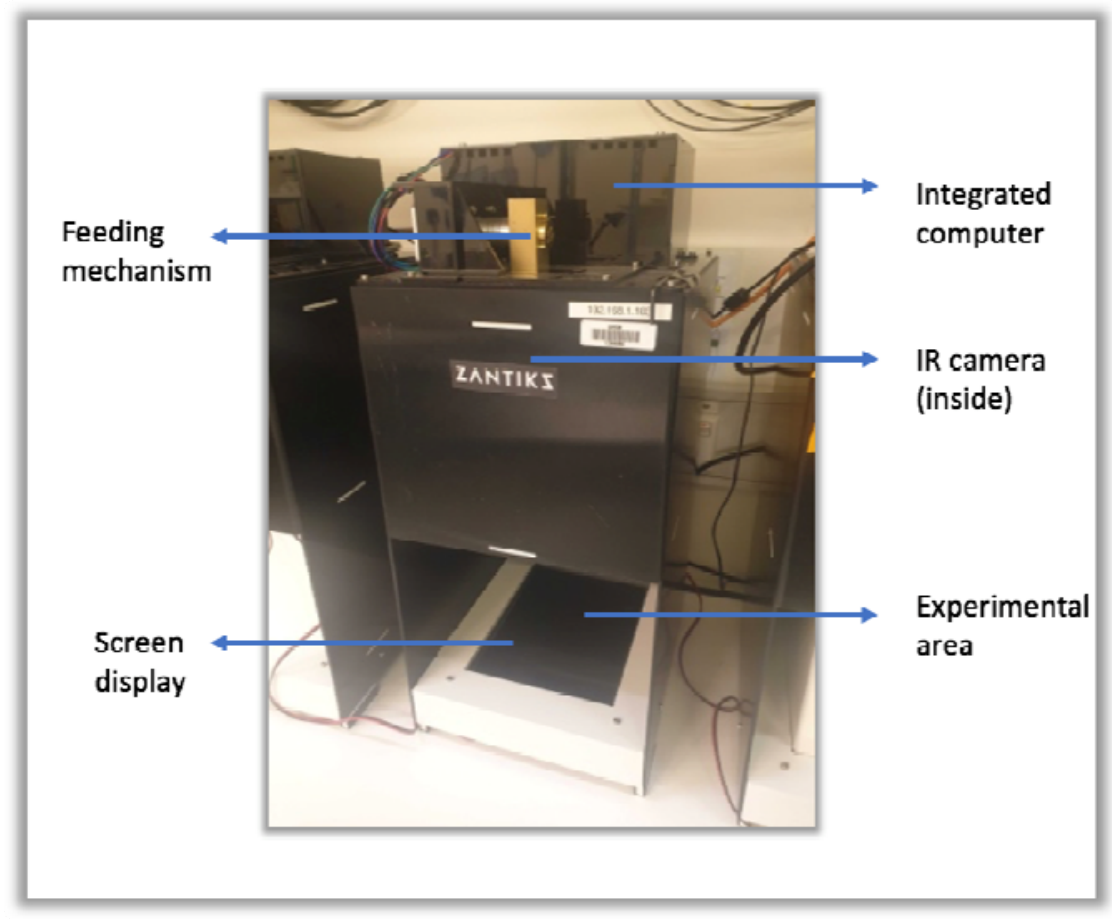

\section{Supplementary Figure 1}

124 Zantiks AD unit. Fully automated experimental box with tracking (IR camera), recording

125 (integrated computer interfaced via console, see Supplementary Figure 2E below) among

126 other capabilities with an open compartment where the assay was conducted, with a screen

127 that holds the tank with experimental subjects during trials. 

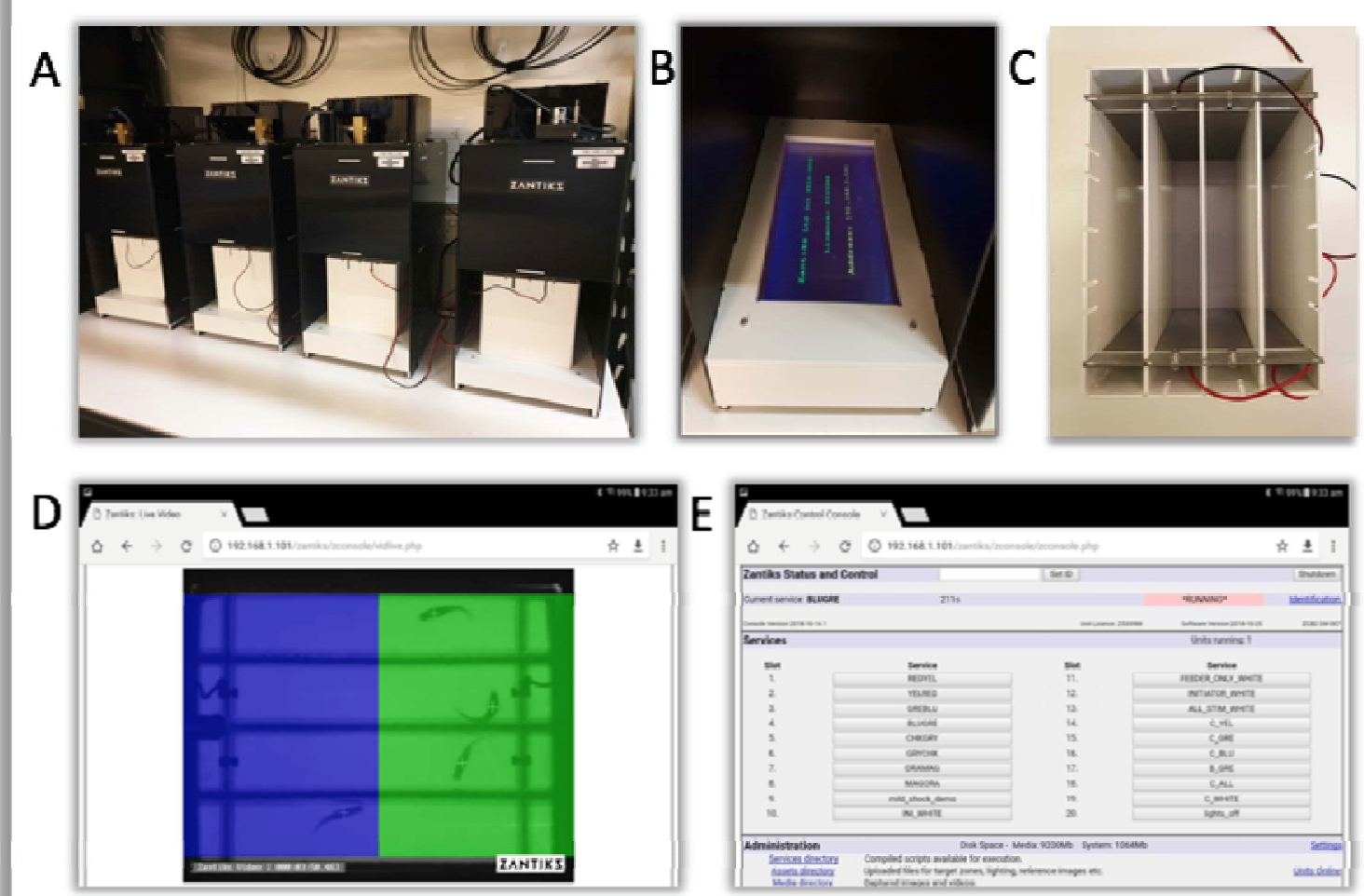

\section{Supplementary Figure 2}

130 Automated conditioning setup. A: All four Zantiks AD experimental units. B: Stimulus screen

131 programmed to present a variety of colours, patterns and images. C: Tank organised for

132 aversive experiment with 4 lanes and 2 mild electric shock plates. D: View of example assay,

133 depicting fish tracking and overlay of perimeters (CS zones). E: Control console for ease of

134 interface with the AD units. 


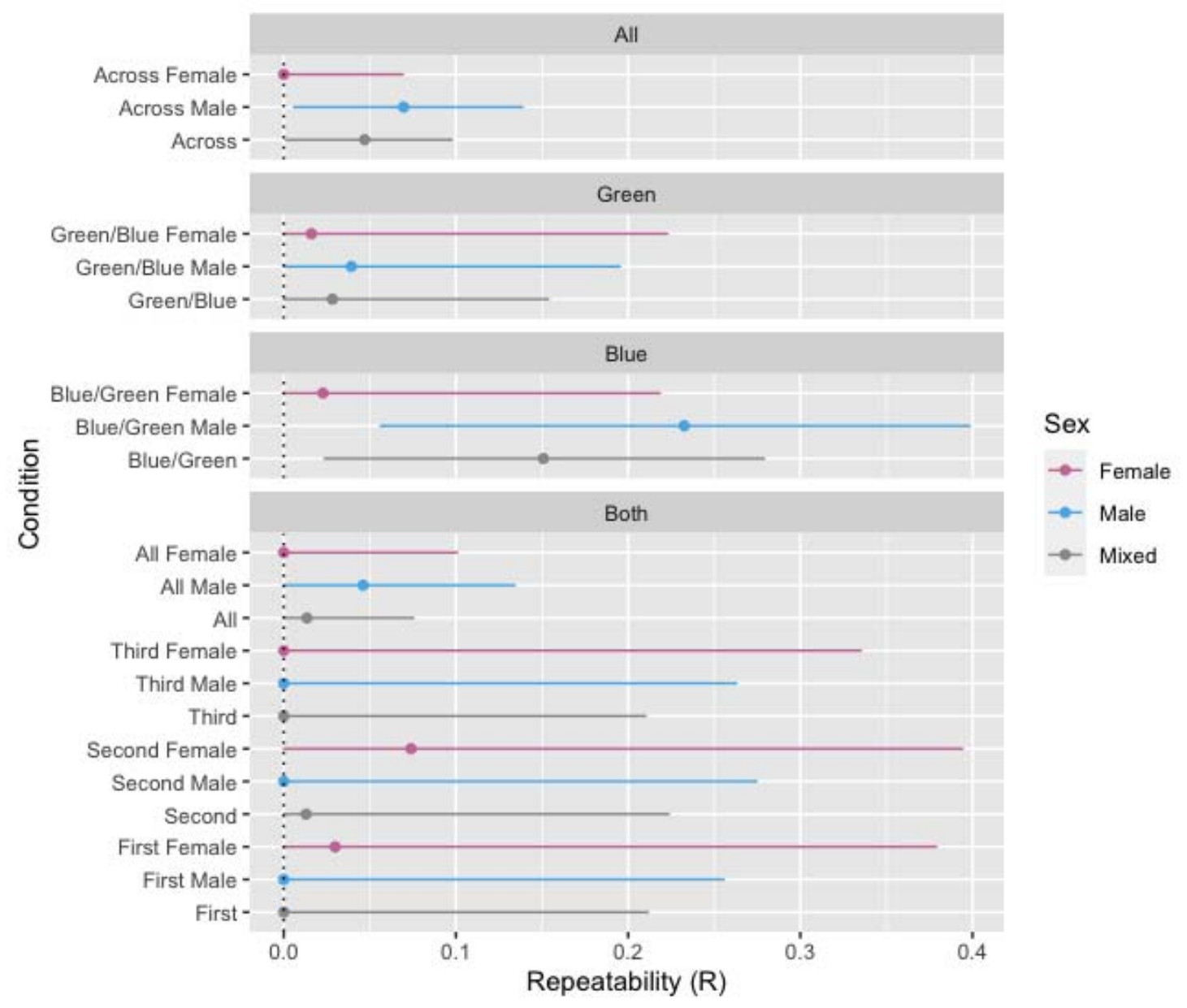

\section{Supplementary Figure 3}

137 The repeatability $(R)$ of aversive learning with $95 \%$ CIs in females, males and both sexes

138 together (mixed). The segments are from top to bottom: Across all conditions, the

139 'Green/Blue' condition, the 'Blue/Green' condition, both 'Green/Blue' and 'Blue/Green'

140 combined. In the bottom segments, the conditions are split into four measurement sets: 'First',

141 the first measurements of both 'Green/Blue' and 'Blue/Green' (set of two measurements),

142 'Second', the second measurements of both 'Green/Blue' and 'Blue/Green', Third, the third

143 measurements of both 'Green/Blue' and 'Blue/Green', 'All', all measurements sets combined

144 (total of 6 measurements). 


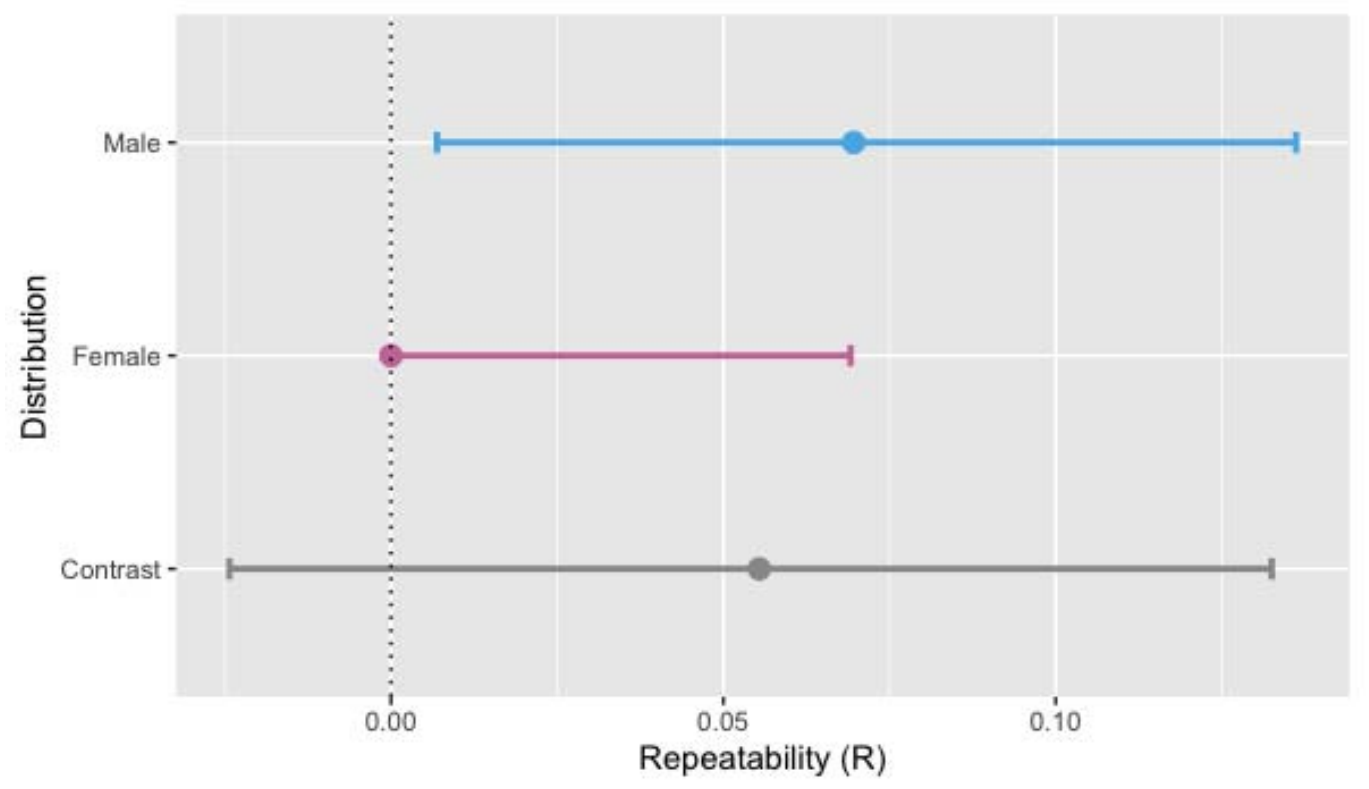

145

\section{Supplementary Figure 4}

147 Male and female zebrafish contrast analysis of repeatability estimates in across conditions

148 trials. Male and females differ in the repeatability bootstrap distribution, however, the contrast

149 analysis indicates by way of the distributions overlapping zero that males and females do not

150 significantly differ in repeatability. 


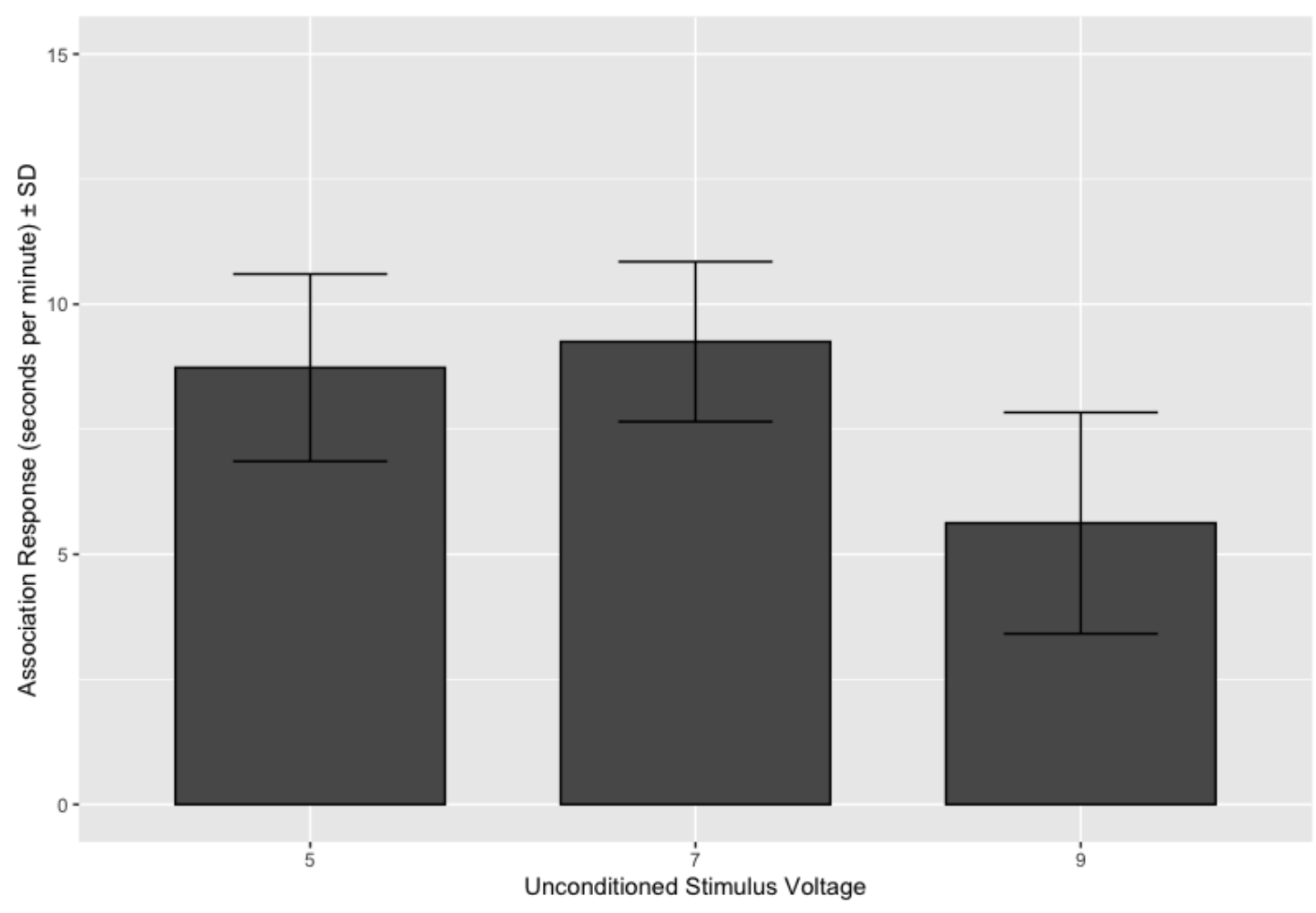

151

\section{Supplementary Figure 5}

153 Zebrafish learning performance in three voltage settings during pilot experiment with

154 standard deviation. Mean difference in CS+ avoidance between baseline and probe phase in 155 seconds per minute in five, seven and nine volts. 
All Conditions

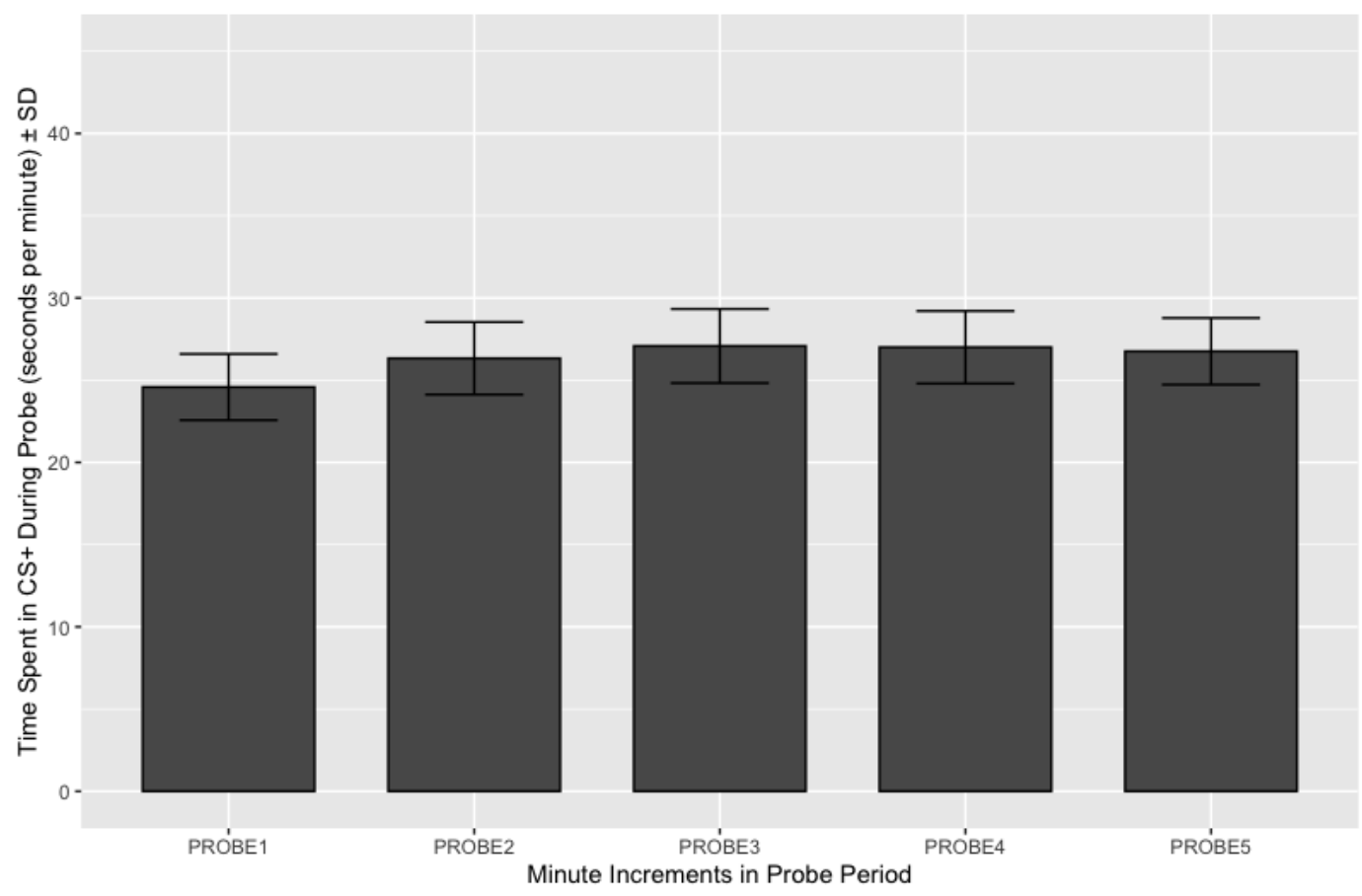

156

157

\section{Supplementary Figure 6}

159 Zebrafish learning performance across all conditions in the probe period with standard

160 deviation. Avoidance of the CS+ is shown separately for each minute of the probe. 

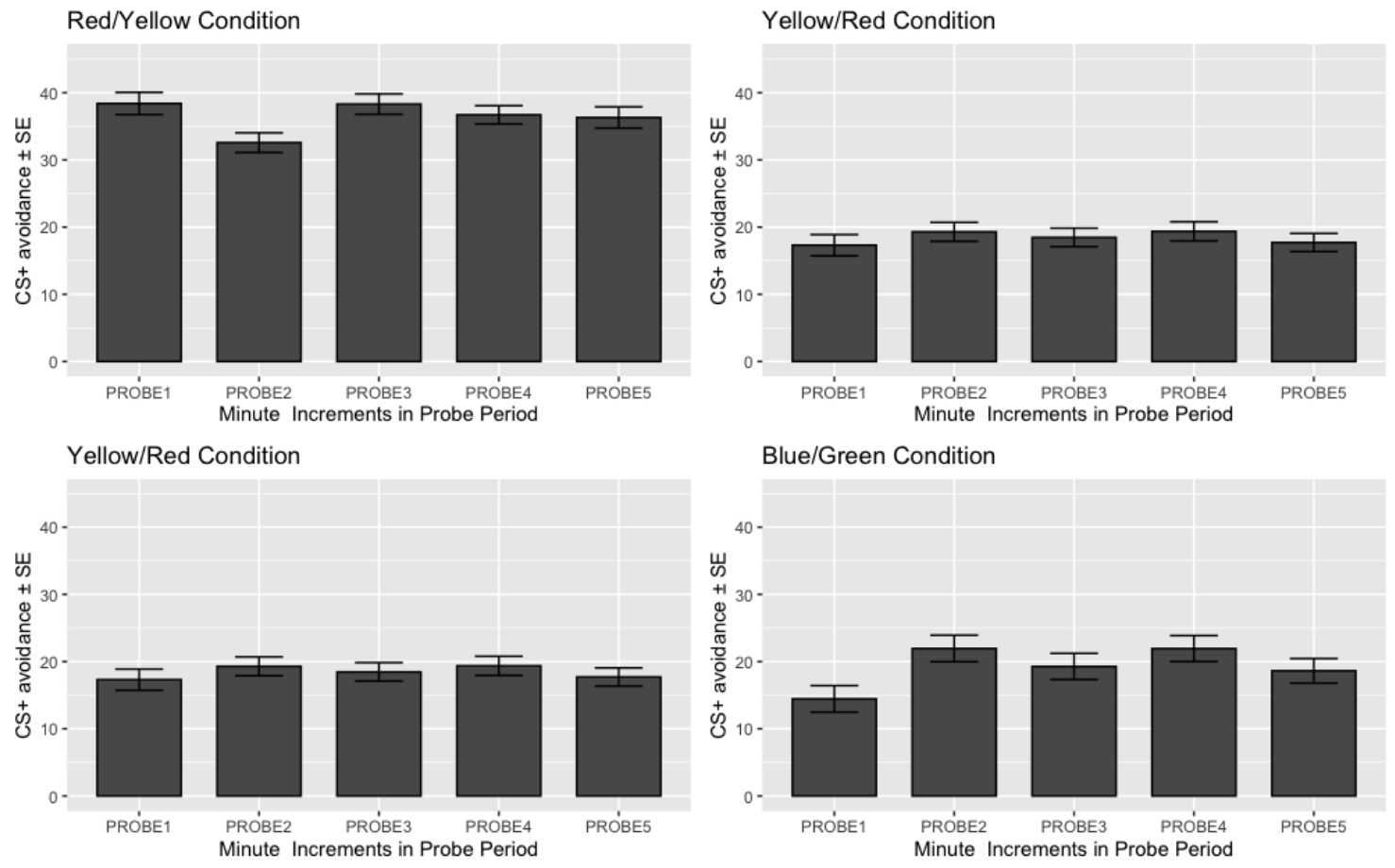

Check/Grey Condition

\section{Grey/Check Condition}
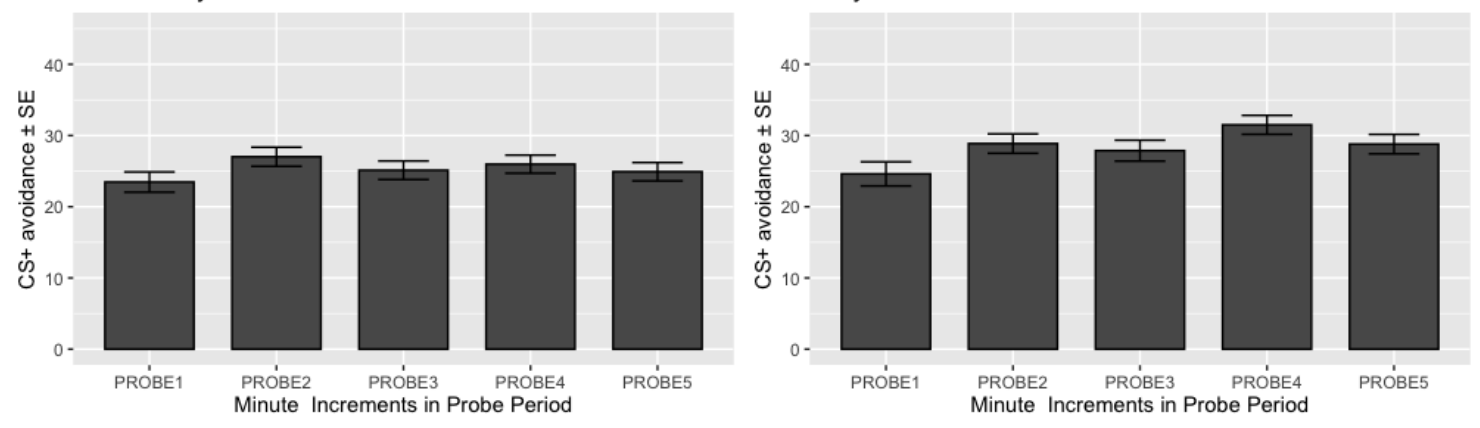

Orange/Magenta Condition
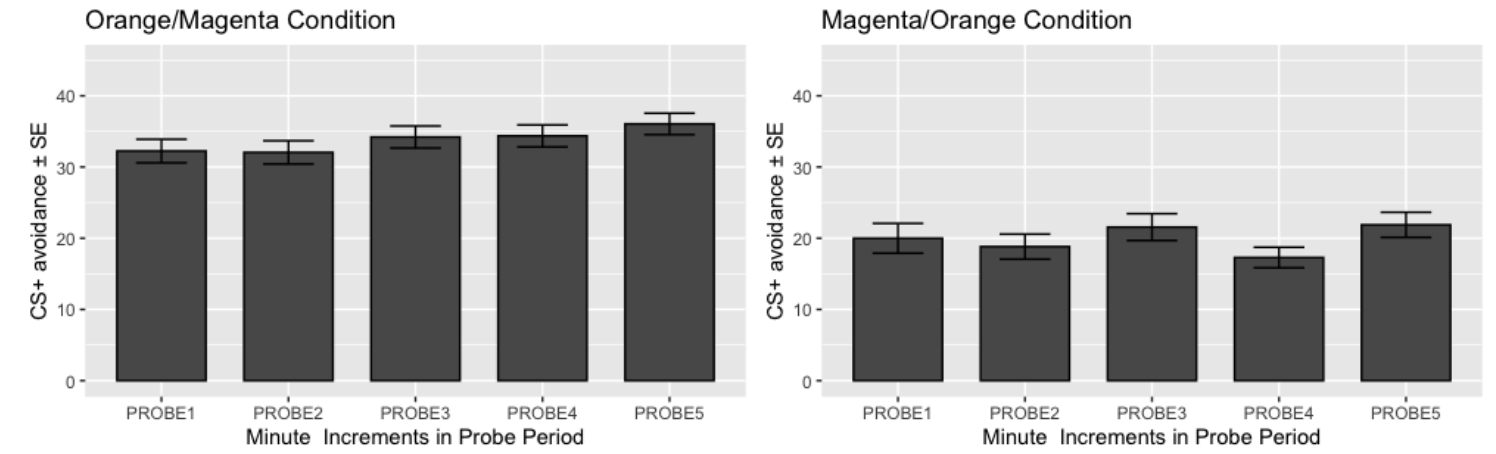

\section{$163 \quad$ Supplementary Figure 7}

164 Zebrafish learning performance in each condition during the probe period with standard error.

165 Avoidance of the CS+ is shown separately for each minute of the probe. 


\section{Supplementary References}

167 Baker, M. R., \& Wong, R. Y. (2019). Contextual fear learning and memory differ between

168 stress coping styles in zebrafish. Scientific Reports, 9(1). https://doi.org/10.1038/s41598-019-

$169 \quad 46319-0$

170 Bell, A. M., Hankison, S. J., Laskowski, K. L., Bell, Alison M, Shala J. Hankison, and K. L.

171 L., Bell, A. M., Hankison, S. J., \& Laskowski, K. L. (2009). The repeatability of behaviour: a

172 meta-analysis. Animal Behaviour, 77(4), 771-783.

173 https://doi.org/10.1016/j.anbehav.2008.12.022.Bell

174 Brock, A. J., Sudwarts, A., Daggett, J., Parker, M. O., \& Brennan, C. H. (2017). A fully

175 automated computer based Skinner box for testing learning and memory in zebrafish.

176 BioRxiv, 110478. https://doi.org/10.1101/110478

177 Fontana, B D, Cleal, M., Clay, J. M., \& Parker, M. O. (2019). Zebrafish (Danio rerio)

178 behavioral laterality predicts increased short-term avoidance memory but not stress-reactivity

179 responses. Animal Cognition. https://doi.org/10.1007/s10071-019-01296-9

180 Kaneko, S., Masuda, R., \& Yamashita, Y. (2019). Memory retention capacity using two

181 different training methods, appetitive and aversive learning, in juvenile red sea bream

182 Chrysophrys major. Journal of Fish Biology, 94(2), 231-240.

183 https://doi.org/10.1111/jfb.13876

184 Kenney, J. W., Scott, I. C., Josselyn, S. A., \& Frankland, P. W. (2017). Contextual fear

185 conditioning in zebrafish. Learning and Memory, 24(10), 516-523.

186 https://doi.org/10.1101/lm.045690.117

187 Namekawa, I., Moenig, N. R., \& Friedrich, R. W. (2018). Rapid olfactory discrimination

188 learning in adult zebrafish. Experimental Brain Research, 236(11), 2959-2969.

189 https://doi.org/10.1007/s00221-018-5352-x

190 Thomson, H., Lamb, S., Besson, A., \& Johnson, S. (2020). Long $\square$ term repeatability of

191 behaviours in zebrafish (Danio rerio). Ethology, 10.1111/eth.13038

192 